\title{
NOTICIAS
}

\section{Grandes desafíos de la salud global}

La salud de los países en desarrollo podría superar algunos problemas si la iniciativa conocida como "Grandes desafíos de la salud global" tiene éxito; este proyecto está basado en el reconocimiento de que la pobreza de salud es uno de los grandes impedimentos para el desarrollo internacional.

Hasta ahora no se había presentado un esfuerzo sistemático para la identificación de los problemas científicos más críticos en materia de salud global, ni se habían destinado fondos en búsqueda de su solución. Esta iniciativa dirigirá sus recursos de manera sustancial y cuidadosa hacia el apoyo de investigaciones en salud pertinentes a los países en desarrollo. Para ello, el pasado 17 de octubre se dieron a conocer los primeros 14 desafíos científicos mundiales en esta materia, en los cuales se centrará su actividad.

La Fundación para los Institutos Nacionales de Salud de los Estados
Unidos de Norteamérica, FNIH por sus siglas en inglés, conjuntamente con la Fundación Bill \& Melinda Gates, hicieron este anuncio en espera de recibir propuestas de investigación de científicos y técnicos talentosos de todo el mundo que puedan contribuir a formular soluciones prácticas y posibles a estos problemas de salud de los países en desarrollo. Se cuenta con el apoyo financiero de 200 millones de dólares destinado por la Fundación Bill \& Melinda Gates para el financiamiento de las operaciones de esta importante proposición.

La iniciativa Grandes desafíos está dirigida por un comité científico internacional presidido por el laureado con el premio Nobel, doctor Harold Varmus, quien es presidente del Memorial Sloan-Kettering Cancer Center y director fundador de los NIH. El comité científico conformado por 20 expertos de 13 países, incluyendo varios de los países en desarrollo, estuvieron encargados de definir los 14 desafíos para lo cual se apoyaron en la aportación de más de 1000 ideas presentadas por científicos de 75 países.

Los 14 desafíos anunciados están asociados con siete metas:

- Mejoramiento del sistema de vacunación de la niñez

- Creación de nuevas vacunas

- Control de insectos transmisores de enfermedades

- Mejoramiento de la nutrición para promoción de la salud

- Mejoramiento de los tratamientos médicos en enfermedades infecciosas

- Curas latentes e infecciones crónicas

- Medición confiable y económica de la salud y la enfermedad de países en desarrollo

La FNIH invita a la comunidad científica a presentar proyectos de investigación en torno a estos desafíos. Para mayor información sobre la iniciativa Grandes desafíos, así como acerca de instrucciones para el envío de propuestas, consulte la página www. grandchallengesgo.org. 